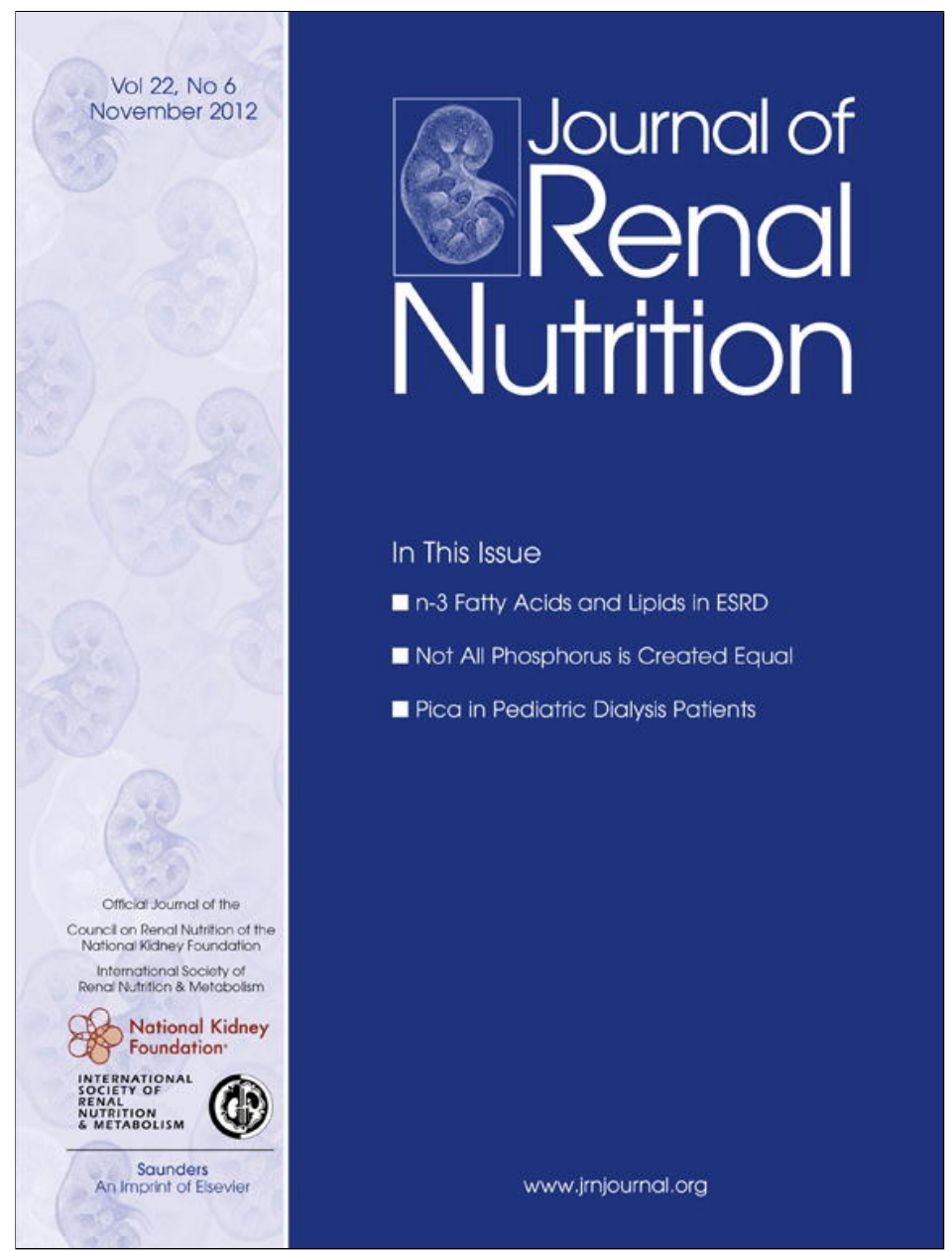

This article appeared in a journal published by Elsevier. The attached copy is furnished to the author for internal non-commercial research and education use, including for instruction at the authors institution and sharing with colleagues.

Other uses, including reproduction and distribution, or selling or licensing copies, or posting to personal, institutional or third party websites are prohibited.

In most cases authors are permitted to post their version of the article (e.g. in Word or Tex form) to their personal website or institutional repository. Authors requiring further information regarding Elsevier's archiving and manuscript policies are encouraged to visit:

http://www.elsevier.com/copyright 


\title{
Nutritional Knowledge in Hemodialysis Patients and Nurses: Focus on Phosphorus
}

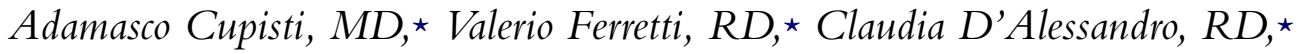 \\ Isabella Petrone, MD, $\dagger$ Adriana Di Giorgio, RD, $\neq$ Mario Meola, MD,* \\ Vincenzo Panichi, MD, $\ddagger$ Paolo Conti, MD, $\$$ Alberto Lippi, MD, $\uparrow$ \\ Raffaele Caprioli, MD, $\Phi$ and Alessandro Capitanini, MD†
}

Objective: To assess the knowledge of adult hemodialysis patients and nurses working in dialysis units, specifically with regard to knowledge of phosphorus and other nutrients related to dietary management of end-stage renal disease.

Design: Cross-sectional cohort study.

Setting: Hemodialysis unit.

Subjects: One hundred ninety-one hemodialysis patients and 105 dialysis nurses, as well as 86 control hospital employees who are not health professionals.

Intervention: Nutritional knowledge was assessed by a 25 -item chronic kidney disease knowledge assessment tool for nutrition, which includes 15 questions on phosphorus and 10 questions on protein, sodium, and potassium knowledge.

Results: The scores obtained by patients were much lower than those of nurses $(11.6 \pm 3.9$ vs. $16.0 \pm 2.2, P<.001)$ but slightly higher than those of controls $(10.6 \pm 3.2, P<.05)$. Patients with phosphorus serum level $>5.5 \mathrm{mg} / \mathrm{dL}$ showed chronic kidney disease knowledge assessment tool for nutrition scores similar to those of patients with a serum phosphorus level $<5.5 \mathrm{mg} / \mathrm{dL}$. The prevalence of right answers to questions regarding knowledge of phosphorus was lower than that regarding knowledge of the other nutrients, both for patients $(38.4 \% \pm 17.8 \%$ vs. $57.3 \% \pm$ $19.9 \%, P<.001)$ and nurses $(55.6 \% \pm 11.1 \%$ vs. $74.8 \% \pm 11.7 \%, P<.001)$ as well as for controls $(30.7 \% \pm 14.5 \%$ vs. $60.1 \% \pm 17.4 \%, P<.001)$.

Conclusions: Our study suggests that nutritional knowledge of hemodialysis patients, although higher than the general population, is lower for phosphorus with respect to the other nutrients, such as protein, sodium, and potassium. This occurs even in patients with hyperphosphatemia or those taking phosphate binder medications. Nurses showed the best scores; however, improvement is necessary, especially with regard to knowledge of phosphorus. Training programs on nutrition for nurses and on information for patients should be implemented. They can contribute to achievement of a more effective control of phosphate balance, reduction of costs, and improvement of the quality of care for hemodialysis patients.

๑ 2012 by the National Kidney Foundation, Inc. All rights reserved.

$\mathrm{H}$ YPERPHOSPHATEMIA IS AN independent risk factor of cardiovascular morbidity and mortality in chronic kidney disease (CKD) patients. Although standard renal replacement

\footnotetext{
^Nephrology Section, Department of Internal Medicine, University of Pisa, Pisa, Italy.

†Nephrology and Dialysis Unit, Pescia Hospital (Pistoia), Pescia, Italy.

$\ddagger$ Nephrology and Dialysis Unit, Versilia Hospital, Versilia, Italy. Italy.

$\$$ Nephrology and Dialysis Unit, Grosseto Hospital, Grosseto,

INephrology and Dialysis Unit, A.O.U. Pisana, Pisa, Italy.
}

therapy represents a life-saving treatment, its effect on the removal of phosphorus remains insufficient to maintain a neutral phosphate balance in wellnourished patients. Thus, the tendency toward
Financial Disclosure: The authors declare that they have no relevant financial interests.

Address reprint requests to Adamasco Cupisti, MD, Nephrology Section, Department of Internal Medicine, University of Pisa, Via Roma 67, 56126 Pisa, Italy.E-mail: acupisti@med.unipi.it

(1) 2012 by the National Kidney Foundation, Inc. All rights reserved.

$1051-2276 / \$ 36.00$

doi:10.1053/j.jrn.2011.11.003 
a positive phosphate balance is the basis for hyperphosphatemia, secondary hyperparathyroidism, increased risk of soft-tissue calcification, hospitalization, and cardiovascular disease. ${ }^{1-5}$

The mainstay treatment of hyperphosphatemia in patients on maintenance dialysis is the use of phosphate binder medications. However, their effectiveness is greatly reduced by an uncontrolled dietary intake of phosphorus. To limit dietary phosphorus load, renal patients need education and information about known dietary sources of phosphorus and especially about the so-called hidden phosphorus (i.e., from the phosphoruscontaining additives in foods). ${ }^{6-8}$ Dialysis patients are usually given dietary instructions regarding food choices to maintain an adequate supply of protein and energy and to reduce dietary intake of phosphorus, sodium, potassium, and fluids. Despite this, nutritional knowledge of phosphorus compared with knowledge of other nutrients was poor in dialysis patients, as assessed by a 25-item CKD knowledge assessment tool for nutrition (CKDKAT-N). ${ }^{9}$

The high morbidity and mortality risk associated with disorders of mineral metabolism makes improving the serum phosphate control in dialysis patients a major goal for nephrologists and renal dietitians. Among the hemodialysis professionals, nurses play an important role because they are near the patients, are more receptive to patients' concerns, and can provide appropriate suggestions, advice, or recommendations. ${ }^{10,11}$

The objective of this study was to assess the knowledge of hemodialysis patients and nurses working in dialysis units, specifically with regard to knowledge of phosphorus and other nutrients related to dietary management of end-stage renal disease.

\section{Subjects and Methods}

Patients receiving thrice-a-week maintenance hemodialysis in 5 dialysis centers in Tuscany (Italy) were asked to enter the study. We assumed psychiatric illness, acute illness, cachexia, and blindness as exclusion criteria. All the subjects and patients who denied their informed consent or who were not collaborative were also excluded. Dialysis nurses were recruited in the same dialysis centers as the patients.

A total of 191 dialysis patients (119 male, 72 female; age, $62 \pm 13$ years) and 105 dialysis nurses (28 male, 77 female; age, $41.0 \pm 7.0$ years) entered the study. A group of 86 hospital employees (26 male, 60 female; age, $48.3 \pm 11.0$ years) who were not health professionals formed a control group.

Data including age, gender, body weight, and use of phosphate binders were collected. Blood samples for biochemical examinations were drawn from the arterial line before the beginning of the first dialysis of the week; serum calcium, phosphorus, potassium, albumin, and urea were assayed, and hemoglobin, spKt/V, and nPNA levels were calculated.

Some features of the studied patients with or without overt hyperphosphatemia are reported in Table 1.

Table 1. Characteristics of the Studied Population of Hemodialysis Patients, and Questionnaires Scores, Stratified by Phosphorus Serum Levels

\begin{tabular}{lccr}
\hline Phosphorus Serum Level & $\begin{array}{c}>5.5 \mathrm{mg} / \mathrm{dL} \\
\mathrm{n}=76\end{array}$ & $\begin{array}{c}<5.5 \mathrm{mg} / \mathrm{dL} \\
\mathrm{n}=106\end{array}$ & $P$ \\
\hline Age, years & $60.1 \pm 14.7$ & $66.7 \pm 11.6$ & $<.001$ \\
Body mass index, $\mathrm{kg} / \mathrm{m}^{2}$ & $26.5 \pm 5.5$ & $24.5 \pm 4.3$ & .012 \\
Dry weight, $\mathrm{kg}$ & $76.1 \pm 18.9$ & $68.5 \pm 15.0$ & .003 \\
Interdialytic weight gain, kg & $2.9 \pm 1.1$ & $2.5 \pm 1.3$ & .128 \\
Serum phosphorus, $\mathrm{mg} / \mathrm{dL}$ & $6.8 \pm 1.1$ & $4.2 \pm 0.8$ & $<.001$ \\
Serum calcium, mg/dL & $8.8 \pm 0.8$ & $8.8 \pm 0.6$ & .781 \\
Ca $\times$ P, mg $/ \mathrm{dL}^{2}$ & $59.9 \pm 11.4$ & $36.8 \pm 8.2$ & .001 \\
Serum potassium, mEq/L & $5.2 \pm 0.8$ & $5.1 \pm 0.7$ & .265 \\
Serum albumin, g/dL & $3.59 \pm 0.46$ & $3.50 \pm 0.41$ & .074 \\
Hemoglobin, g/dL & $11.3 \pm 1.1$ & $11.0 \pm 1.3$ & .661 \\
Kt/V & $1.32 \pm 0.32$ & $1.34 \pm 0.30$ & .002 \\
nPNA, g/kg/day & $1.06 \pm 0.27$ & $0.95 \pm 0.25$ & .275 \\
Questionnaire score & & & .307 \\
$\quad$ Phosphorus (15) & $6.0 \pm 2.7$ & $5.6 \pm 2.6$ & \\
Sodium, potassium, protein (10) & $5.7 \pm 2.0$ & $6.0 \pm 1.8$ & \\
\hline
\end{tabular}


The nutritional knowledge was evaluated using the CKDKAT-N. ${ }^{9}$ This questionnaire includes 25 multiple-choice questions reflecting knowledge of 4 nutrients that are relevant for dialysis patients' nutrition: 15 questions concern phosphorus and the remaining 10 test knowledge with regard to protein, sodium, and potassium.

Test answers to each subset of questions related to the knowledge of phosphorus, sodium, potassium, and protein were recorded, and composite scores of knowledge of phosphorus and of the other nutrients were calculated. The score used for statistical analysis was equal to the number of correct responses.

\section{Statistical Analysis}

Descriptive statistics is given as mean \pm standard deviation. Statistical analysis was performed by one-way analysis of variance, and the Bonferroni test was used for post hoc analysis.

Pearson correlation test was performed. Differences were considered as statistically significant at $P<.05$.

\section{Results}

Figure 1 shows that patients' scores were much lower than those of nurses $(11.6 \pm 3.9$ vs. 16.0 $\pm 2.2, P<.001)$, although patients slightly outperformed the controls $(10.6 \pm 3.2, P<.05)$.

In all 3 groups, the percentage of correct answers for phosphorus knowledge was lower when compared with knowledge of the other nutrients (Fig. 2).

Patients with phosphorus serum level $>5.5$ $\mathrm{mg} / \mathrm{dL}$ showed CKDKAT-N scores similar to those of patients with a serum phosphorus level



Figure 1. CKDKAT-N scores obtained by patients, nurses, and controls.

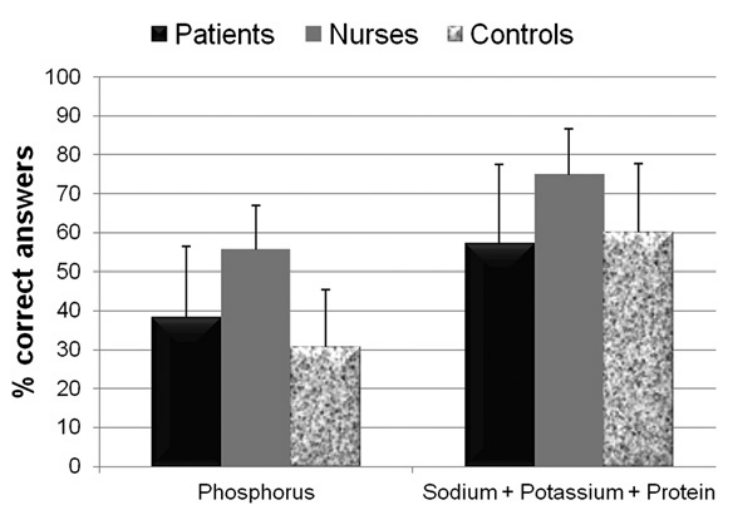

Figure 2. Percentage of correct answers to questions regarding the knowledge of phosphorus and other nutrients for patients, nurses, and controls.

$<5.5 \mathrm{mg} / \mathrm{dL}$; as expected, the former were younger and ate more proteins than the latter (Table 1).

Regarding the prescription of phosphate binders, patients were divided into 2 groups: the first $(\mathrm{n}=110)$ was prescribed sevelamer and/or lanthanum carbonate, and the second $(n=81)$ assumed no phosphate binders or a low dose of calcium. The former showed a significantly higher phosphate score than the latter $(6.2 \pm 2.6$ vs. $5.0 \pm$ $2.6, P<.001)$, but the average percentage of correct answers was low in both groups $(39.7 \%$ and $36.5 \%$, respectively).

No difference was found between males and females in any of the groups. In the patients' group, no relationship was found between CKDKAT-N scores and biochemistry data, in particular with regard to serum phosphorus, calcium $\times$ phosphorus product, albumin, or body weight. Meanwhile, a significant inverse relationship was found between phosphorus score and age $(r=-0.363$, $P<.001)$ but not between age and the scores of the other group of 10 questions.

Dialysis duration was $73 \pm 75$ months. No relationship was found between dialysis vintage and CKDNAT-N scores (total, phosphorus, or nonphosphorus score). Nurses have been working with dialysis patients for $124 \pm 66$ months; no association was found between this period and CKDNAT-N scores.

Finally, when patients older than 60 years were excluded from the analysis, so that the age was similar to that of nurses and control subjects, CKDKAT-N scores for phosphorus knowledge in this subgroup including 60 patients $(6.9 \pm 2.4)$ remained significantly higher than in controls 
$(4.6 \pm 2.2, P<.001)$ and lower than in nurses $(8.3 \pm 1.6, P<.001)$.

\section{Discussion}

Our study suggests that patients on hemodialysis have poor phosphorus knowledge as compared with other nutrients of interest for renal patients. This was also the case for patients with hyperphosphatemia and for those taking phosphate binders. However, when compared with the general population, a higher score was observed even when the groups were matched for age. This may be due to the general dietary instruction usually given during predialysis and dialysis management care.

Hyperphosphatemia is a major risk factor of death in CKD patients. Poor control of serum phosphorus persists despite the implementation of phosphate binders as standard therapy in dialysis patients. Dietary phosphate restriction is mandatory for patients with $\mathrm{CKD},{ }^{12,13}$ who have a tendency toward positive phosphate balance. In fact, phosphorus retention is a main physiopathological factor leading to secondary hyperparathyroidism, vascular calcification, and increased cardiovascular morbidity and mortality. ${ }^{1,2}$ Despite the widespread use of phosphate binder medications, restriction of phosphorus intake is needed to obtain close control of serum phosphorus. This is of crucial importance not only for CKD patients but also for cardiac patients and even for the general population. In fact, evidence exists that an increase of serum phosphate, even within the normal range, is associated with increased cardiovascular risk in cardiac patients and in the general population. $^{14,15}$

Dietary counseling should be routinely used to educate patients regarding phosphate sources and the consequences of high serum phosphorus and to reinforce adherence. Some studies have evaluated the usefulness of educational counseling in the control of phosphatemia in dialysis patients. As a whole, they suggest that focused education programs are effective in enhancing knowledge about phosphate in the dialysis population, and the impact may be most marked in patients with poor baseline knowledge. ${ }^{16-20}$

In addition, the modern diet and food industry have made it more difficult for patients to avoid foods rich in phosphorus. Several additives contain phosphate, which is almost completely absorbed by the gastrointestinal tract, representing a dangerous extraphosphate $\operatorname{load}^{21-23}$ originating from food and beverages.

Nutritional therapy is an essential tool for management care of patients with CKD. Many nutritional parameters need to be monitored, including energy and protein (quality and quantity), mineral (sodium, potassium, calcium, phosphorus), and fluid intake. Dietary phosphorus management remains particularly difficult and complex in CKD patients. It has been recently underlined that dietary manipulation is an underappreciated tool for reducing serum phosphorus levels. ${ }^{24}$ Patients' education by renal dietitians remains a major component of the effort to control serum phosphorus, and restriction of dietary phosphorus intake is needed to allow a successful phosphate binder action. ${ }^{23-25}$ Unfortunately, our findings show that there is little education of the patients by a dietitian in the dialysis unit, as the dietitian is rarely available.

Many studies about dietary management in dialysis patients enhance the importance of nutritional education to prevent malnutrition and to minimize complications of end-stage renal disease. European Best Practice Guidelines on nutrition in hemodialysis patients ${ }^{13}$ reported that dietary education improves phosphate control and that intensive counseling on the phosphate content of food reduces serum phosphate and calcium phosphate product. However, there are no specific recommendations regarding how to implement an education plan for patients. The initiative to organize counseling interventions is left to the nephrologists and dietitians; if the dietitian is not present, once again the role of a trained nurse appears decisive. Hemodialysis nurses may play an important role in the reinforcement of adherence and in educating patients regarding the consequences of high serum phosphorus and the importance of dietary phosphorus restriction. In our series, nurses showed a better nutritional knowledge as compared with patients or the general population, but nutritional knowledge regarding phosphorus seemed poor. Moreover, in our series, nurses are not required to take at least 1 university nutrition course in their training/education, but nutrition is only incorporated into aspects of their program.

Increased training in nutrition given to nurses and better information given to patients with poor control of serum phosphate may facilitate a more effective budget control, reduction of costs, 
and improvement of the quality of care for hemodialysis patients. ${ }^{26}$

A multidisciplinary team including nephrologists, renal dietitians, and dialysis nurses can help to improve the nutritional status and to ensure adequate dietary intakes. In particular, dialysis nurses provide a crucial benefit by understanding the relationship between nutrition parameters and the possible complications and complexities of the daily nutritional challenges of dialysis patients. If an adequate nutritional knowledge is achieved, the question "So just what can I eat?" can be answered with greater confidence. ${ }^{27}$

\section{Practical Application}

The practical application of this study consists in the implementation of cyclical education plans with practical application experiences for patients (e.g., group classes with various foods for patients to select or "rate" their phosphorus levels). Group meetings may be useful in encouraging patients to "discuss" their daily life dietary problems and putting the patients at greater ease when they are unsure of information but afraid to ask for guidance from the staff or physician.

Nursing continuing education on nutrition should also be implemented in the dialysis departments; nephrologists and renal dietitians should take courses, training, and seminars focused on knowledge of food composition and on information addressed to reduce the effective dietary phosphorus load. Greater attention to dietary education in dialysis patients and professionals will contribute to a more effective control of phosphorus balance, reduction of costs, and increase of the quality of care of hemodialysis patients.

\section{References}

1. Mathew S, Tustison KS, Sugatani T, Chaudhary LR, Rifas L, Hruska KA. The mechanism of phosphorus as a cardiovascular risk factor in CKD. J Am Soc Nephrol. 2008;19:1092-1105.

2. Young B, Sherrard DJ, Andress DL. Serum phosphate levels and mortality risk among people with chronic kidney disease. J Am Soc Nephrol. 2005;16:520-528.

3. Noori N, Kalantar-Zadeh K, Kovesdy CP, Bross R, Benner D, Kopple JD. Association of dietary phosphorus intake and phosphorus to protein ratio with mortality in hemodialysis patients. Clin J Am Soc Nephrol. 2010;5:683-692.

4. Galetta F, Cupisti A, Franzoni F, et al. Left ventricular function and calcium phosphate plasma levels in uraemic patients. J Intern Med. 2005;258:378-384.
5. Cozzolino M, Ciceri P, Volpi EM, Olivi L, Messa PG. Pathophysiology of calcium and phosphate metabolism impairment in chronic kidney disease. Blood Purif. 2009;27:338-344.

6. Shaw-Stuart NJ, Stuart A. The effect of an educational patient compliance program on serum phosphate levels in patients receiving hemodialysis. J Ren Nutr. 2000;10:80-84.

7. Reddy V, Symes F. Dietitian-led education program to improve phosphate control in a single-center hemodialysis population. J Ren Nutr. 2009;19:314-320.

8. Morey B, Walker R, Davenport A. More dietetic time, better outcome? A randomized prospective study investigating the effect of more dietetic time on phosphate control in end-stage kidney failure hemodialysis patients. Nephron Clin Pract. 2008; 109:c173-c180.

9. Pollock JB, Faffery JB. Knowledge of phosphorus compared with other nutrients in maintenance dialysis patients. J Ren Nutr. 2007; 17:323-328.

10. Kalantar-Zadeh K, Gutekunst L, Mehrotra R, et al. Understanding sources of dietary phosphorus in the treatment of patients with chronic kidney disease. Clin J Am Soc Nephrol. 2010;5:519-530.

11. Cupisti A, Morelli E, D'Alessandro C, Lupetti S, Barsotti G. Phosphate control in chronic uremia: don't forget diet. J Nephrol. 2003;16:29-33.

12. Barsotti G, Cupisti A. The role of dietary phosphorus restriction in the conservative management of chronic renal disease. J Ren Nutr. 2005;15:189-192.

13. Fouque D, Vennegoor M, ter Wee P, et al. EBPG guideline on nutrition. Nephrol Dial Transplant. 2007;22(suppl 2): ii45-ii87.

14. Tonelli M, Sacks F, Pfeffer M, Gao Z, Curhan G. Relation between serum phosphate level and cardiovascular event rate in people with coronary disease. Circulation. 2005;112:2627-2633.

15. Dhingra R, Sullivan LM, Fox CS, et al. Relations of serum phosphorus and calcium levels to the incidence of cardiovascular disease in the community. Arch Intern Med. 2007;167: $879-885$.

16. Ford JC, Pope JF, Hunt AE, Gerald B. The effect of diet education on the laboratory values and knowledge of hemodialysis patients with hyperphosphatemia. J Ren Nutr. 2004;14: 36-44.

17. Schlatter S, Ferrans CE. Teaching program effects on high phosphorus levels in patients receiving hemodialysis. ANNA J. 1998;25:31-36.

18. Caldeira D, Amaral T, David C, Sampaio C. Educational strategies to reduce serum phosphorus in hyperphosphatemic patients with chronic kidney disease: systematic review with meta-analysis. J Ren Nutr. 2011;21:285-294.

19. Cupisti A, D’Alessandro C, Baldi R, Barsotti G. Dietary habits and counseling focused on phosphate intake in hemodialysis patients with hyperphosphatemia. J Ren Nutr. 2004;14: 220-225.

20. Park KA, Choi-Kwon S, Sim YM, Kim SB. Comparison of dietary compliance and dietary knowledge between older and younger Korean hemodialysis patients. J Ren Nutr. 2008; 18:415-423.

21. Uribarri J, Calvo MS. Hidden sources of phosphorus in the typical American diet: does it matter in nephrology? Semin Dial. 2003;16:186-188.

22. Sherman RA, Mehta O. Dietary phosphorus in dialysis patients: potential impact of processed meat, poultry, 
and fish products as protein sources. Am J Kidney Dis 2009;54:18-23.

23. Benini O, D’Alessandro C, Gianfaldoni D, Cupisti A. Extra-phosphate load from food additives in commonly eaten foods: a real and insidious danger for renal patients. J Ren Nut. 2011;21:303-308.

24. Daugirdas JT. Proposed controlled trials of phosphate reduction in CKD: which whey should we go? Kidney Int. 2010;77:929-930.
25. Poduval RD, Wolgemuth C, Ferrell J, Hammes MS. Hyperphosphatemia in dialysis patients: is there a role for focused counseling? J Ren Nutr. 2003;13:219-223.

26. Knapp S. Bone metabolism and disease-K/DOQI impact on nurses and dietitians. Nephrol Nurs J. 2004;31: 333-335

27. Beto JA, Nicholas M. So just what can I eat? Nutritional care in patients with diabetes mellitus and chronic kidney disease. Nephrol Nurs J. 2009;36:497-504. 